\title{
Using PacBio sequencing to investigate the bacterial microbiota of traditional Buryatian cottage cheese and comparison with Italian and Kazakhstan artisanal cheeses
}

Hao Jin, Lanxin Mo, Lin Pan, Qaingchaun Hou, Chuanjuan Li, laptueva Darima, and Jie Yu ${ }^{1}$

Key Laboratory of Dairy Biotechnology and Engineering, Key Laboratory of Dairy Products Processing, Inner Mongolia Agricultural University, Hohhot 010018, P.R. China

\begin{abstract}
Traditional fermented dairy foods including cottage cheese have been major components of the Buryatia diet for centuries. Buryatian cheeses have maintained not only their unique taste and flavor but also their rich natural lactic acid bacteria (LAB) content. However, relatively few studies have described their microbial communities or explored their potential to serve as LAB resources. In this study, the bacterial microbiota community of 7 traditional artisan cheeses produced by local Buryatian families was investigated using single-molecule, real-time sequencing. In addition, we compared the bacterial microbiota of the Buryatian cheese samples with data sets of cheeses from Kazakhstan and Italy. Furthermore, we isolated and preserved several LAB samples from Buryatian cheese. A total of $62 \mathrm{LAB}$ strains (belonging to 6 genera and 14 species or subspecies) were isolated from 7 samples of Buryatian cheese. Full-length $16 \mathrm{~S}$ rRNA sequencing of the microbiota revealed 145 species of 82 bacterial genera, belonging to 7 phyla. The most dominant species was Lactococcus lactis (43.89\%). Data sets of cheeses from Italy and Kazakhstan were retrieved from public databases. Principal component analysis and multivariate ANOVA showed marked differences in the structure of the microbiota communities in the cheese data sets from the 3 regions. Linear discriminant analyses of the effect size identified 48 discriminant bacterial clades among the 3 groups, which might have contributed to the observed structural differences. Our results indicate that the bacterial communities of traditional artisan cheeses vary depending on geographic origin. In addition, we isolated novel and valuable LAB resources for the improvement of cottage cheese production.
\end{abstract}

Received January 6, 2018.

Accepted April 4, 2018.

${ }^{1}$ Corresponding author: yujie8301@sina.com
Key words: bacterial diversity, Russia, single molecule, real time, traditional artisan cheese

\section{INTRODUCTION}

Buryatia, a federal subject of Russia belonging to the Siberian Federal District, is located in the south of Eastern Siberia along Lake Baikal. Traditional fermented dairy products have become an indispensable part of the Russian diet and life. Since ancient times, traditional Russian dairy products have mainly included cottage cheeses (soft cheese, milk tofu), butter, yogurt, sour cream, fermented dairy drinks, and kefir (Tsydenova, 2008). In fact, each nation has its own unique culture and customs, as well as its own types and methods of making dairy products (Kamber, 2007; Litopoulou-Tzanetaki and Tzanetakis, 2014). In general, cottage cheeses are made by first storing raw milk in porcelain or casks for natural fermentation for approximately $3 \mathrm{~d}$. Then the fermented milk is transferred to a pot and cooked slowly on the stove with continuous stirring until it becomes thick. To improve the sensory quality of the final product, cream or butter is usually added to the mixture before allowing it to ripen. Finally, the mixture is shaped into a mold. The ripening process involves many natural microorganisms. The complex microbial communities are mainly from the raw material and the direct environment in which the cheeses are made, such as the utensils and containers for cheese production. As a part of Russia, Buryatia has preserved its regional and traditional cottage cheeses.

The physical properties and sensory quality of cheeses are consequences of fermentation by a complex natural microbial community (Escobar-Zepeda et al., 2016). The consumption of raw milk cheese has positive effects on enteric microbiota and immunity (Koning et al., 2008; Montel et al., 2014). High-throughput sequencing has been applied to investigate the microbial communities present in ecological environments, including food products (Ercolini, 2013). In particular, this method 
allows rapid and accurate identification of microorganisms, including those that are difficult to culture and those present in low abundance (Walsh et al., 2017). This approach has been used to study the bacterial diversity of various cheeses. Quigley et al. (2012) analyzed the microbiological compositions of 62 Irish soft, semi-hard, and hard cheeses using high-throughput sequencing techniques based on cheese type, milk, and production techniques. The results showed that the microbial structure varied by cheese type, animal milk source, additives used, and whether the milk was pasteurized. Other traditional cheeses have also been studied, such as Latin-style cheese, Kazakhstan cheese, Italian cheese, and Mexican cheese (Lusk et al., 2012; González-Córdova et al., 2016; Li et al., 2017; Silvetti et al., 2017). However, the microbial communities present in Buryatian cheeses have yet to be fully explored.

The third-generation DNA sequencer, PacBio RS II (Pacific Biosciences, Menlo Park, CA), is an advanced DNA sequencing platform that produces sequences of long read lengths and a low degree of bias. This technique is useful for generating microbiota profiles based on full-length $16 \mathrm{~S}$ rRNA gene sequences at a high taxonomic resolution at the species level (Nakano et al., 2017). Earlier sequencing technologies, such as 454-pyrosequencing and the Illumina platform, provided sequence information based only on the partial 16S rRNA gene, which limited the taxonomic resolution. Singer et al. (2016) compared the results of microbiota profiling of samples taken from meromictic Sakinaw Lake using the PacBio SMRT sequencing and Illumina platform. PacBio SMRT sequencing resulted in less ambiguous classification while allowing wider species diversity identification. Thus, the PacBio SMRT platform could be used to describe the microbial communities more accurately with higher phylogenetic resolution (Singer et al., 2016). This method has successfully been applied to evaluate the safety of infant formula, based on the bacterial population of traditional fermented koumiss and milk products enhanced with probiotics (Gesudu et al., 2016; Zheng et al., 2016; Xu et al., 2017). However, few studies have applied the PacBio SMRT sequencing technology to study the microbiota composition of traditional cheeses, with the exception of a previous study that depicted the bacterial profiles in artisan cheese from Kazakhstan (Li et al., 2017).

With the modernization of society and the industrialization of cheese making, conventional homemade cheese production has declined. As the Buryatia region still maintains its traditional methods and starter strains in cheese production, we anticipated a high microbial richness and diversity in cheese samples collected from local households. Common to other fermented foods, lactic acid bacteria (LAB) are the most important bacteria responsible for the sensory quality and physical properties of traditional fermented dairy products (Yu et al., 2012). These bacteria can serve as valuable resources for future improvements in the production of cheeses and other fermented products. Thus, we characterized the microbiota communities of cheese samples collected in Buryatia based on full-length 16S rRNA profiling using PacBio technology. We isolated LAB from a variety of samples with the aim of preserving these valuable microbial resources for subsequent research and industrial application. Moreover, we compared the bacterial microbiota of Buryatia cheese with those from Kazakhstan and Italy using the data sets of 18 Kazakhstan and Italian cheeses retrieved from public databases.

\section{MATERIALS AND METHODS}

\section{Samples}

A total of 7 traditional fermented cheeses were obtained from 7 different farms in Buryatia, represented as BL1 to BL7. All farms produced traditional cottage cheeses using similar methods. The samples were collected using a sterile spoon, stored in sealed bags, and sent to our laboratory packed on ice. Upon delivery to the laboratory, samples were stored at $-80^{\circ} \mathrm{C}$ and analyzed within a few days of collection. Data sets from 18 cheese samples from Italy and Kazakhstan were retrieved for comparative analyses (Table 1; De Filippis et al., 2014).

\section{Enumeration and Isolation of $L A B$}

One gram of sample was serially diluted with $9 \mathrm{~mL}$ of saline $(0.85 \% \mathrm{NaCl})$ for each gradient. The pour plate method was used to determine the bacterial load in the cheese samples. A total of $200 \mu \mathrm{L}$ of diluted sample was plated onto de Man, Rogosa, and Sharpe (MRS, Difco Laboratories, Detroit, MI) and M17 (Oxoid Ltd., Waltham, MA) agar plates. The inoculated plates were cultured in an anaerobic incubator at $37^{\circ} \mathrm{C}$ for 48 to 72 h. Colonies were counted and grouped based on colony morphology, including color, shape, and size. Representative single colonies were streaked and propagated on the corresponding culture medium. All isolates were tested for catalase activity and Gram staining. Grampositive and catalase-negative isolates were recultivated for further identification. Meanwhile, the isolates were kept at $-40^{\circ} \mathrm{C}$ for long-term storage.

\section{Identification of $L A B$}

Genomic DNA of the selected isolates was extracted based on previously reported method (Yu et al., 2009). 


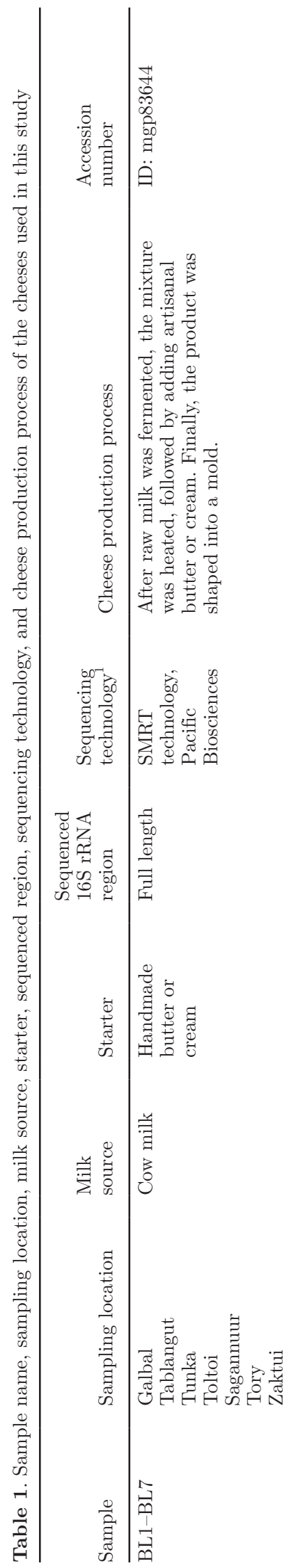

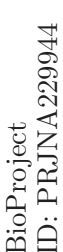

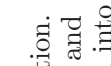

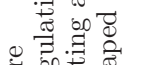

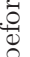

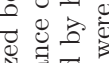

菏跤

范进

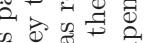

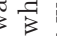

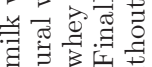

层要运

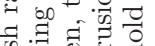

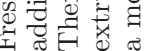

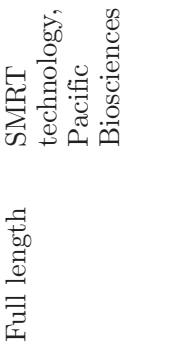

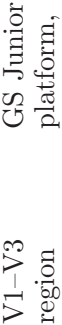

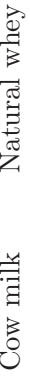

皇

สี. ⿷ี

莺客落

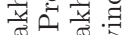

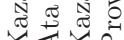

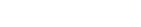

进电

昰题

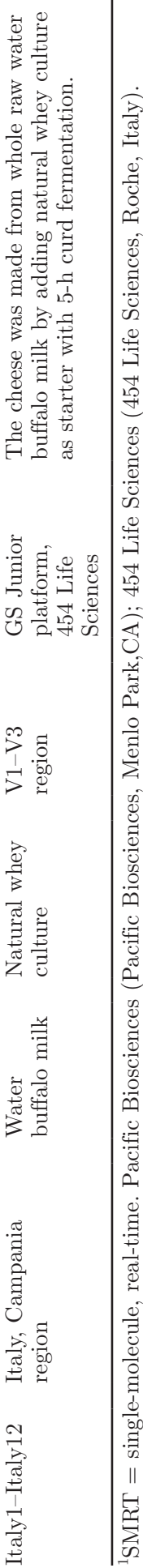

The 16S rRNA gene was amplified on a thermal cycler (PTC-200, MJ Research, Waltham, MA) using the purified extracted genomic DNA as templates and the universal primer pair 27F (5'-AGAGTTTGATCCTGGCTCAG-3') and 1495R (5'-CTACGGCTACCTTGTTACGA-3'). Each sample contained $1 \times$ Taq buffer (including $1.5 \mathrm{mM} \mathrm{MgCl}$, TaKaRa Bio Inc., Shiga, Japan), $0.2 \mu M$ deoxynucleotide triphosphates, $10 \mathrm{pmol}$ of each primer, $10 \mathrm{ng}$ of bacterial template, and $1.0 \mathrm{U}$ Ex Taq polymerase (TaKaRa Bio Inc.). The reaction conditions were as follows: $94^{\circ} \mathrm{C}$ for $5 \mathrm{~min}, 94^{\circ} \mathrm{C}$ for 1 $\min , 55^{\circ} \mathrm{C}$ for $1 \mathrm{~min}, 72^{\circ} \mathrm{C}$ for $2 \mathrm{~min}, 30$ cycles, and then $72^{\circ} \mathrm{C}$ for $10 \mathrm{~min}$. The DNA sequencing was performed by Majorbio Bio-Pharm Technology Corporation Limited (Shanghai, China). A phylogenetic tree was constructed based on the generated sequences using the MEGA version 6.0 software (http://www.megasoftware .net).

\section{Sample Preparation and DNA Extraction for PacBio SMRT Sequencing}

Cheese samples collected from different parts of the same cheese were mixed and ground into powder. Total DNA was extracted from $2 \mathrm{~g}$ of cheese powders using a modified cetyltrimethylammonium bromide method (Doyle, 1987). The quality of the extracted DNA was checked by agarose gel electrophoresis on a $0.8 \%$ gel and spectrophotometry (optical density at $260 \mathrm{~nm} / 280$ nm ratio, Thermo Fisher Scientific, Waltham, MA). All DNA samples were stored at $-20^{\circ} \mathrm{C}$ for further experiments.

\section{Amplification of Full-Length 16S rRNA and PacBio SMRT Sequencing}

The forward 27F (5'-GAGAGTTTGATCCTGGCTCAG-3') and the reverse 1541R (5'-AAGGAGGTGATCCAGCCGCA- $3^{\prime}$ ) primers were used to amplify the full-length 16S rRNA gene with the PCRBIO Taq DNA polymerase (PCR Biosystems Ltd., London, UK). The primers contained a set of 16-nucleotide barcodes. The volume of the final reaction mixture was $50 \mu \mathrm{L}$. Each sample contained $1 \times$ PCRBIO reaction buffer, $10 \mathrm{ng}$ of template DNA, $10 \mathrm{pmol}$ of each primer, and $1.0 \mathrm{U}$ of $\mathrm{Taq}$ DNA polymerase. The reaction conditions were as follows: $95^{\circ} \mathrm{C}$ for $5 \mathrm{~min}$, then 30 cycles at $95^{\circ} \mathrm{C}$ for $30 \mathrm{~s}, 58^{\circ} \mathrm{C}$ for $45 \mathrm{~s}$, and $72^{\circ} \mathrm{C}$ for 1 min with a final extension of $72^{\circ} \mathrm{C}$ for $7 \mathrm{~min}$ (Hou et al., 2015). The Agilent DNA 1000 Kit and an Agilent 2100 Bioanalyser (Agilent Technologies, Santa Clara, CA) were used to check the quality of the PCR products. Purified PCR products were used to construct DNA libraries with the Pacific Biosciences Template Prep Kit 2.0 (Pacific 
Biosciences). The P6-C4 chemistry on the PacBio RS II platform was used to sequence the purified amplification products.

\section{Sequence Processing and Bioinformatics Analysis}

Raw data were generated under the protocol RS ReadsOfinsert.1. The QIIME package was used to obtain the high-quality sequences. To extract the highquality sequences, the minimum full passes, minimum predicted accuracy, minimum read lengths of inserts, and maximum read lengths were set to $5,90,1,400$, and 1,800, respectively (Hou et al., 2015). The barcode and primer sequences were removed to create the data set. Data sets of the Italy and Kazakhstan cheeses were also filtered to select for sequences with a read length over 425 bp. Then, PyNAST and UCLUST were applied to align sequences under $100 \%$ clustering of sequence identity to obtain representative sequences. Afterward, sequences were classified into operational taxonomic units (OTU) under the threshold of 97\% through UCLUST (Lozupone et al., 2006). The Ribosomal Database Project II database was used to assign the taxonomy of each OTU representative sequence with an $80 \%$ confidence threshold (Cole et al., 2007). A de novo taxonomic tree was constructed using representative OTU set in FastTree for downstream analysis (Price et al., 2010). The $\alpha$-diversity was evaluated by the Shannon-Wiener, Simpson's diversity, Chao1, and rarefaction estimators. Both weighted and unweighted calculations were applied in the principal component analyses and multivariate ANOVA to analyze the bacterial communities. Different regions were classified by Mahalanobis distance, and the data were analyzed by the Matlab R2016a software (MathWorks, Natick, MA). The Kruskal-Wallis test was used to evaluate any difference in bacterial population between samples. Linear discriminant analysis (LDA) effect size method based on a normalized relative abundance matrix was used to identify the significant differences between cheese samples. The $\mathrm{R}$ package version 3.1.2 (https:// www.r-project.org/) and the Origin software version 8.5 (OriginLab Corporation, Hampton, MA) were used to produce the graphs.

\section{Nucleotide Sequence Accession Number}

All the PacBio SMRT sequencing data reported in this study were deposited at Metagenomic Rapid Annotations using Subsystems Technology database (accession no. mgp83644, http://metagenomics.anl.gov/). All the LAB isolates sequences were submitted to the National Center for Biotechnology Information database under accession numbers MF784166 to MF784228.

\section{RESULTS}

\section{Enumeration and Isolation of $L A B$}

The LAB counts in the samples ranged from 3.47 \pm 0.99 to $7.99 \pm 0.50 \mathrm{log} \mathrm{cfu} / \mathrm{g}$. A total of 62 different isolates were isolated from 7 cheeses collected from Buryatia, of which 34 and 28 were isolated from MRS and M17 medium, respectively. Each strain was presumed to be LAB, based on the gram-positive and catalase-negative phenotype. The isolates were further identified to the species level by $16 \mathrm{~S}$ rRNA gene sequencing. The relationships between representative isolates and strains types were represented using phylogenetic trees (Figures 1 and 2). These isolates were identified as Enterococcus casseliflavus (2 strains), Enterococcus durans (4 strains), Enterococcus faecalis (2 strains), Enterococcus faecium (5 strains), Lactobacillus brevis (3 strains), Lactobacillus delbrueckii ssp. bulgaricus ND02 (1 strain), Lactobacillus fermentum (1 strain), Lactobacillus gasseri (1 strain), Lactobacillus paracasei (2 strains), Lactobacillus plantarum (12 strains), Lactococcus lactis (11 strains), Leuconostoc lactis (2 strains), Leuconostoc pseudomesenteroides (1 strain), Pediococcus acidilactici (4 strains), Pediococcus pentosaceus (4 strains), and Streptococcus thermophilus (7 strains).

\section{Bacterial Community Diversity and Richness in the Buryatian Cheese}

A total of 67,178 high-quality sequences (average of $9,596.85 \pm 2,512.28$ per sample) were obtained from the 7 Buryatian cheese samples, samples containing the most and least sequences were BL4 $(13,387)$ and BL6 (5,851), respectively. A total of 14,710 OTU were obtained (average of 2,101.43 $\pm 1,107.55$ OTU per sample). The number of reads, OTU, observed species (ranging from 751.5 to 1,943.42), Chao1 index (ranged $3,364.03$ to 11,638.1), Simpson index (ranged 0.75 to 0.98 ), and Shannon index (ranged 4.58 to 8.22 ) are shown in Supplemental Table S1 (https://doi.org/10 .3168/jds.2018-14403). The Shannon diversity and the rarefaction curves suggested that the majority of sample bacterial phenotypes had already been captured in the current analysis (Supplemental Figure S1; https:// doi.org/10.3168/jds.2018-14403).

\section{Bacterial Composition of Buryatian Cheese}

Seven bacterial phyla were identified in the sampled cheeses, and the major phyla (Supplemental Table S2, https://doi.org/10.3168/jds.2018-14403, mean relative abundance $>0.1 \%$ ) were Firmicutes $(73.57 \%)$ and 
Proteobacteria (26.27\%). Eighty-two genera (Figure 3a) were identified, the major ones (mean relative abundance $>1 \%$ ) being Lactococcus (51.46\%), Streptococcus (17.81\%), Pseudomonas (5.48\%), Acetobacter (4.83\%), Klebsiella (3.36\%), Lactobacillus (2.36\%), Acinetobacter (1.84\%), and Raoultella (1.63\%). In addition, the bacterial community varied markedly between samples. The dominant genus was Lactococcus in samples BL1-5, whereas the most prevalent genera for samples BL6 and BL7 were Acetobacter (33.71\%) and Streptococcus $(81.85 \%)$, respectively.

A total of 145 species were detected (Figure $3 \mathrm{~b}$ ), the major ones being Lc. lactis $(43.89 \%)$, S. thermophilus (8.52\%), Lactococcus raffinolactis (6.12\%), Acetobacter cibinongensis (4.80\%), Lactobacillus helveticus (1.95\%), Klebsiella pneumonia (1.76\%), Acinetobacter johnsonii (1.28\%), and Klebsiella oxytoca (1.11\%). The bacterial species also highly variable. Lactococcus lactis was predominant in samples BL1 (85.59\%), BL3 (38.41\%), BL4
(39.80\%), and BL5 (77.42\%), whereas Lc. raffinolactis (39.67\%), A. cibinongensis (33.52\%), and S. thermophilus (53.44\%) dominated samples BL2, BL6, and BL7, respectively. Interestingly, BL1 and BL5 contained a high relative abundance of $K$. pneumonia $(6.18 \%)$ and K. oxytoca $(10.11 \%)$.

\section{Comparison of Bacterial Profiles of Cheese from Different Regions}

To evaluate differences between the bacterial microbiota of cheeses from various origins, comparative analyses were performed with the sequences generated in this study and the microbiota data sets of Kazakhstan and Italian cheeses retrieved from public databases. First, the microbial richness and diversity were compared between the cheeses from the 3 regions (Figure 4). Based on the Chao1 index and number of observed species, the Italian cheese contained the highest level of

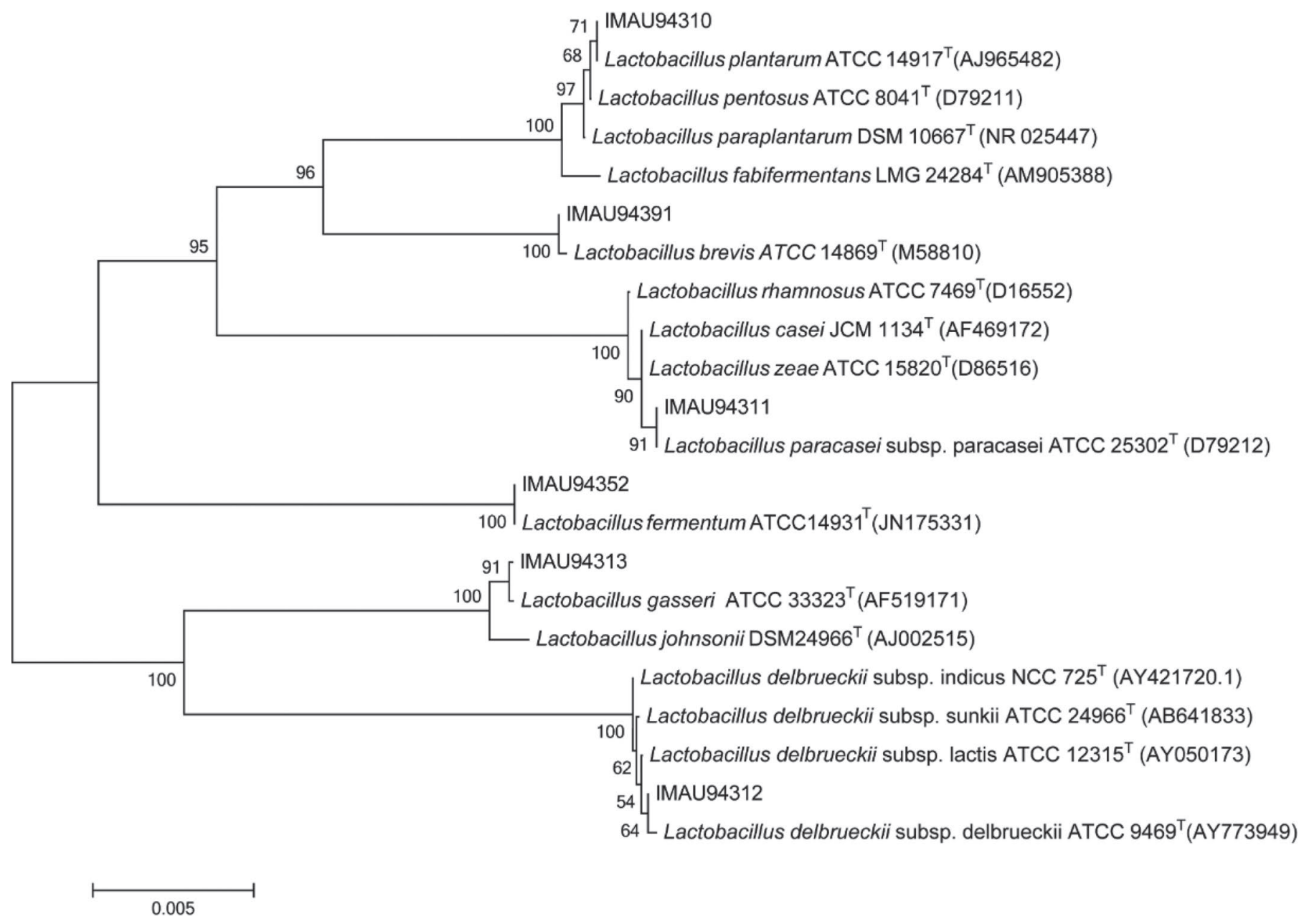

Figure 1. Neighbor-joining tree of $16 \mathrm{~S}$ rRNA genes showing the phylogenetic relationships among 6 Lactobacillus species with the strain type of their related genera. 


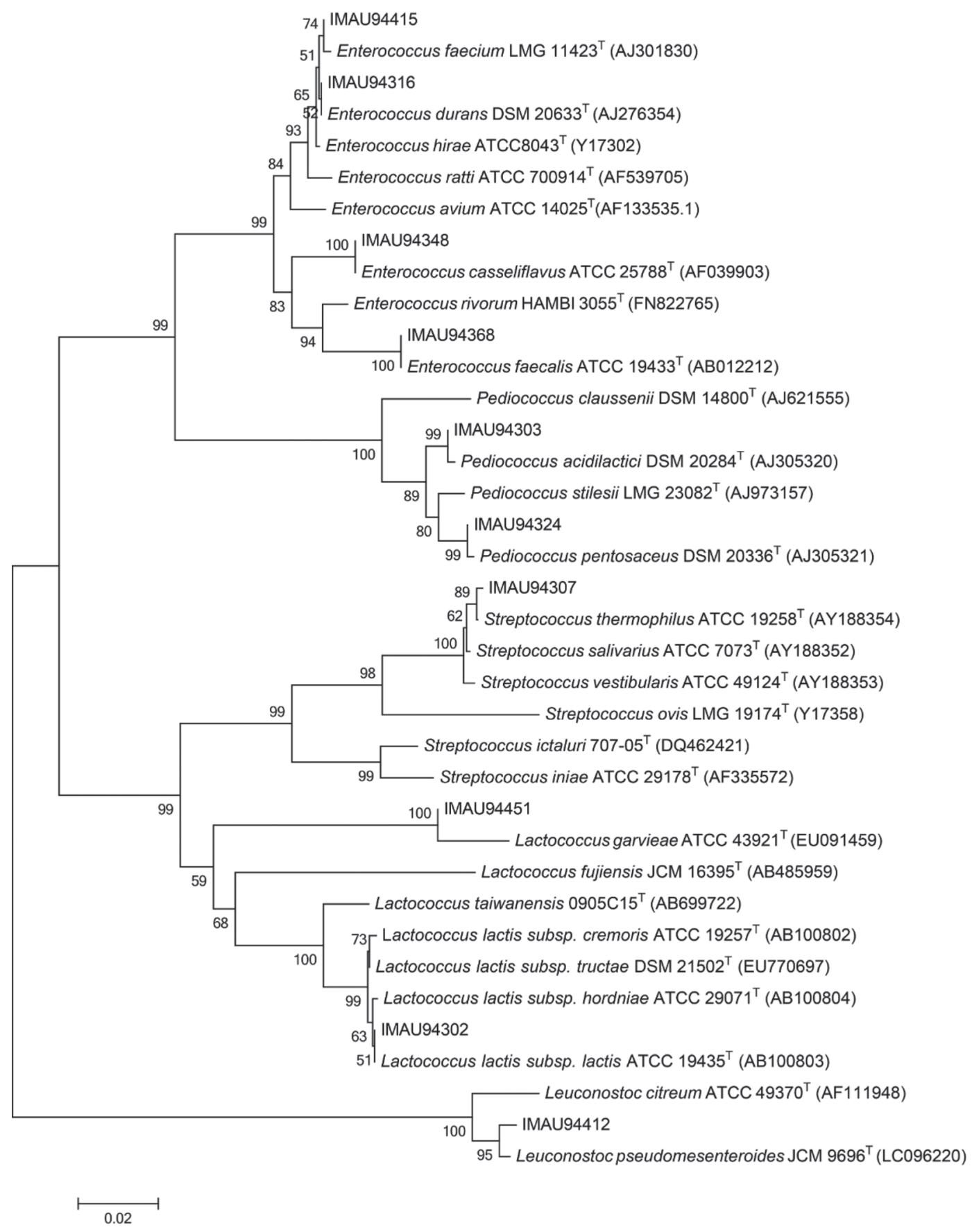

Figure 2. Neighbor-joining tree of $16 \mathrm{~S}$ rRNA genes showing the phylogenetic relationships among 16 isolated cocci with the strain type of their related genera.

bacterial richness. On the other hand, the Kazakhstan cheese had the highest values for the Shannon index and Simpson index, which suggests that the Kazakhstan cheese had the highest level of bacterial diversity.

To visualize differences in the microbiota structure between the 3 sample data sets (Buryatian, Italian, and Kazakhstan), principal component analyses were performed based on the weighted and unweighted UniFrac distances (Figure 5). In general, the symbols representing cheeses from the 3 regions were well separated on both the weighted and unweighted score plots, although mild overlapping was observed between symbols representing the Buryatian and Kazakhstan cheeses on the weighted plot. These results suggest the apparent 
distinction in the microbiota structure among the 3 cheese types. Multivariate ANOVA further supported the existence of significant differences in the microbiota structure among the 3 cheese types $(P<0.001$ in all cases, for both weighted and unweighted analyses).

A total of 13 phyla were identified within the complete data set of the 3 cheese groups. Both the phyla Firmicutes and Proteobacteria had an overall relative abundance $>1 \%$, but that of Firmicutes was significantly higher than that of the latter $(P<0.05)$ and was consistently dominant across all regions (Table 2). The genera Lactobacillus, Streptococcus, and Lactococcus were most prevalent across samples from the 3 regions (Table 2 and Supplemental Table S3, https://doi.org/ 10.3168/jds.2018-14403). To identify differential abundant taxa, LDA of the effect size were performed with an LDA threshold score of 3.5. We identified 48 bacterial clades that significantly differed in relative abundance, including 25 Buryatian taxa, 13 Kazakhstan taxa, and 10 Italian taxa (Figure 6 and Supplemental Table S4, https://doi.org/10.3168/jds.2018-14403).

\section{DISCUSSION}

We isolated and identified 62 LAB strains belonging to 6 genera and 14 species or subspecies. The dominant species was L. plantarum, representing $19.35 \%$ of all isolates, followed by Lc. lactis and S. thermophilus, similar to previous studies on traditional cheeses ( $\mathrm{Yu}$ et al., 2015; Luiz et al., 2017). Viable LAB counts showed variation between individual samples. For example, higher levels of LAB were found in BL1 $(7.77 \pm 1.32$ $\log \mathrm{cfu} / \mathrm{g})$, BL4 $(7.47 \pm 0.46 \log \mathrm{cfu} / \mathrm{g})$, and BL5 (7.99 $\pm 0.50 \log \mathrm{cfu} / \mathrm{g})$ than in BL2 (4.32 $\pm 0.78 \log \mathrm{cfu} / \mathrm{g})$, BL3 (3.47 $\pm 0.99 \log \mathrm{cfu} / \mathrm{g})$, and BL6 (4.46 $\pm 0.58 \mathrm{log}$ $\mathrm{cfu} / \mathrm{g})$. Although similar artisan methods were used to make the cheese samples in this study, subtle differences in the production process might contribute to obvious variation between samples. Several factors may affect the abundance and viability of LAB in the final product, particularly the heating process, raw milk source, and the addition of different ingredients such as cream or butter. The initial starter culture from each household might also differ between households, which could directly change the LAB profile of the final product. More importantly, the different strains might have unequal levels of adaptability toward environmental changes during the cheese-production process.

The taxa obtained using the culture-independent method accounted for most of those obtained by the culture-dependent method; only a few isolates were not detected. It is possible that the bacterial content of the samples was low and insufficient for detection using the current sequencing depth; an increase in sequencing depth would likely detect the bacteria. In addition, the composition of cheese microbiota determined by $16 \mathrm{~S}$

\section{a}

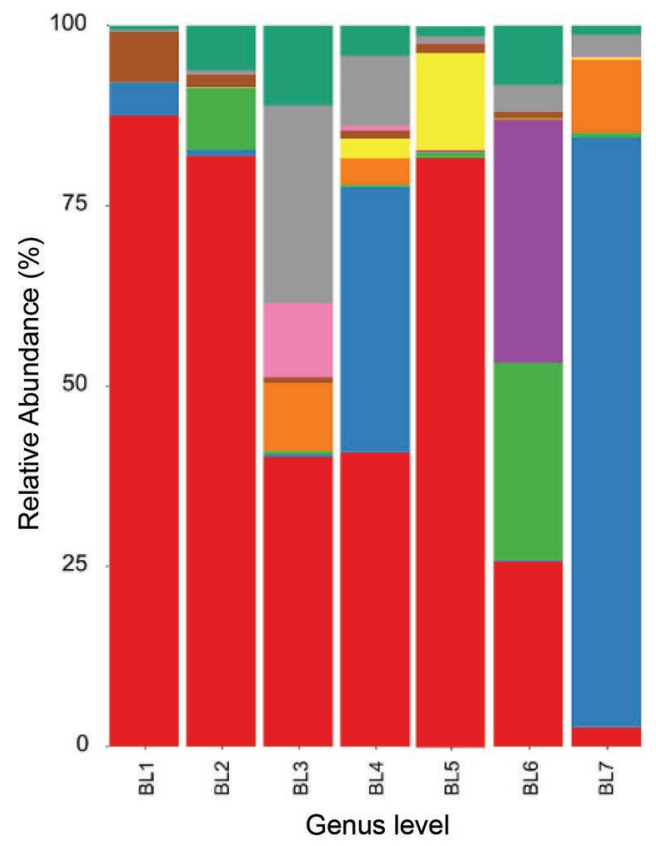

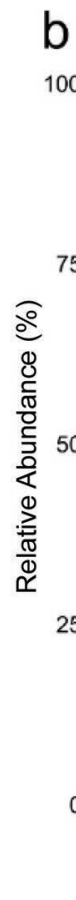

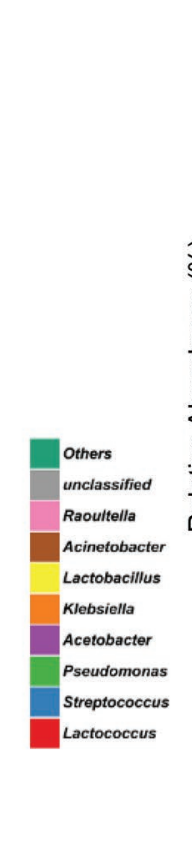

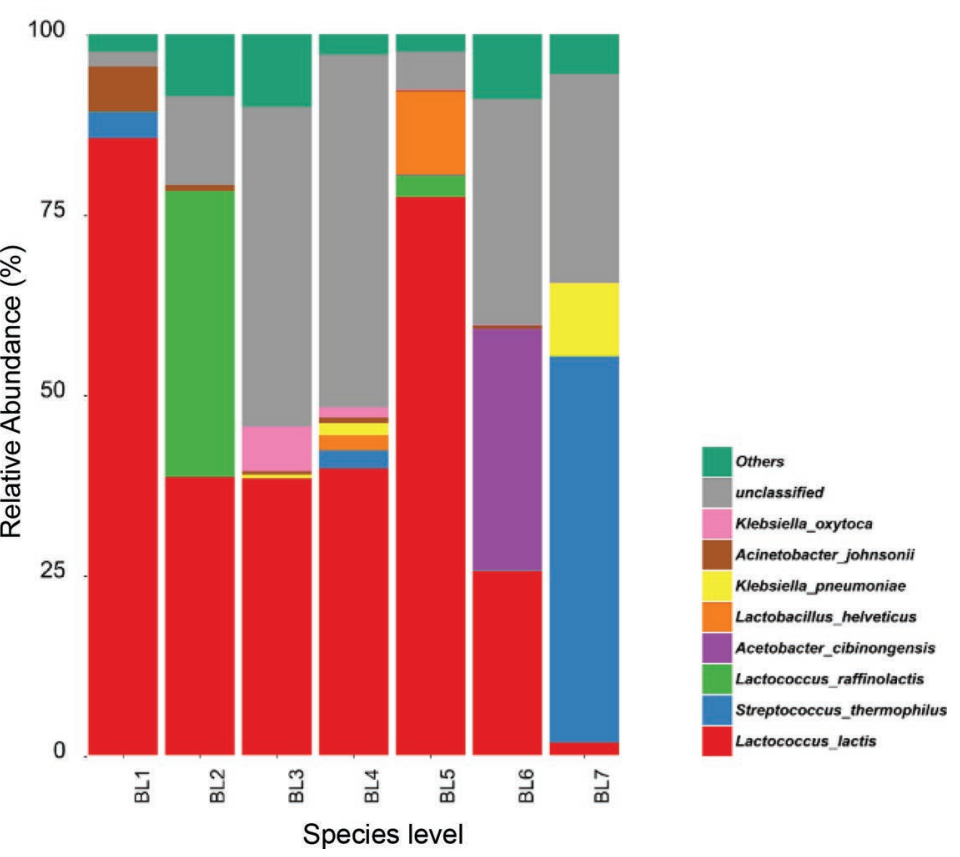

Figure 3. Relative abundances and bacterial diversity of the microbiota of Buryatian cheese (BL1-BL7) at the genus (a) and species (b) levels. Color version available online. 

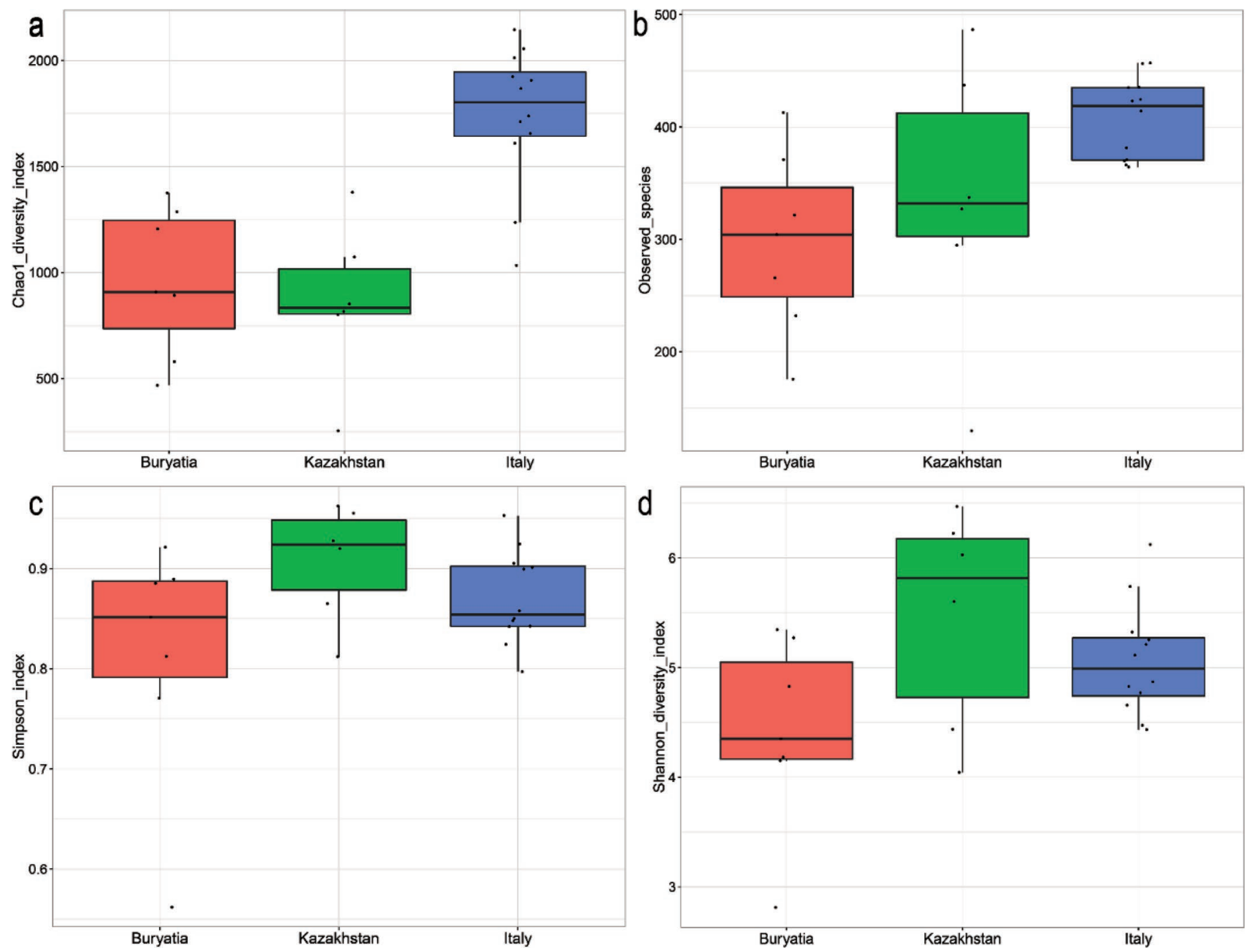

Figure 4. Boxplots of $\alpha$-diversity indexes. Chao1 index (a), number of observed species (b), Simpson index (c), and Shannon index (d) of cheese samples. The whisker caps represent the minimum and maximum values. The box plot middle, upper, and lower lines represent the median value and first and third quartiles, respectively. Filled dots represent each sample. Color version available online.

rRNA profiling revealed great variation among samples. Unlike other traditional cheeses, the manufacturing of Buryatian cheese involves adding butter and cream. Although similar methods were used in cheese production in the households where the cheese samples were collected, the indigenous microflora of the cream or butter (or both) might have differed, leading to the observed difference in the final cheese microbiota composition. It would be interesting to further investigate how the endogenous bacteria in these raw materials shape the composition of the final cheese microbiota. Our results show that the phyla Firmicutes and Proteobacteria were predominant in traditional cheeses, in agreement with previous studies (Riquelme et al., 2015; Dalmasso et al., 2016). Moreover, as the predominant species present in Buryatian cheese, Lc. lactis, is widely used as starter to improve product quality (Fernández et al., 2011), the species not only converts lactose into lactic acid but also contributes to cheese flavor (O'Sullivan et al., 2000). To the best of our knowledge, Lc. raffinolactis and Lc. lactis are new starter cultures that are used in fermented milk because of their interaction during the fermentation process. In this mixed culture, Lc. raffinolactis can compensate for the shortage of slow-growing strains of L. lactis in milk; moreover, $L$. lactis can compensate for the poor caseinolytic activity of Lc. raffinolactis. Strong complementarity between them may be useful for developing dairy products with new properties (Kimoto-Nira et al., 2012). Streptococcus thermophilus is recognized as the most important microorganism in the dairy industry. It improves the ecological environment for lactic acid fermentation and 
Table 2. Major bacterial phyla and genera in the $16 \mathrm{~S}$ rRNA data sets of the cheeses

\begin{tabular}{llclr}
\hline Sample & $\begin{array}{l}\text { Bacterial } \\
\text { phylum }\end{array}$ & $\begin{array}{c}\text { Relative } \\
\text { abundance }\end{array}$ & $\begin{array}{l}\text { Bacterial } \\
\text { genus }\end{array}$ & $\begin{array}{c}\text { Relative } \\
\text { abundance }(\%)\end{array}$ \\
\hline Buryatia & Firmicutes & 74.13 & Lactococcus & 52.17 \\
& Proteobacteria & 25.72 & $\begin{array}{l}\text { Streptococcus } \\
\text { Pseudomonas }\end{array}$ & 17.75 \\
Kazakhstan & & & Lactobacillus & 5.35 \\
& Firmicutes & 92.83 & Lactococcus & 31.53 \\
& Proteobacteria & 6.72 & Streptococcus & 16.39 \\
Italy & & & Lactobacillus & 51.54 \\
& Firmicutes & 99.58 & Streptococcus & 46.34 \\
& Proteobacteria & 0.38 & & 1.62 \\
\hline
\end{tabular}

beneficially lowers the cholesterol levels by inhibiting cholesterol synthase activity (Arioli et al., 2007; Mora and Arioli, 2014). In addition, Lactobacillus rhamnosus is involved in the formation of flavor during cheese rip- ening (Lazzi et al., 2014). Acetobacter cibinongensis can oxidize alcohol or sugars incompletely to form acetic acid. Acetic acid bacteria are widely used in the food industry to produce vinegar, wine, beer, and other
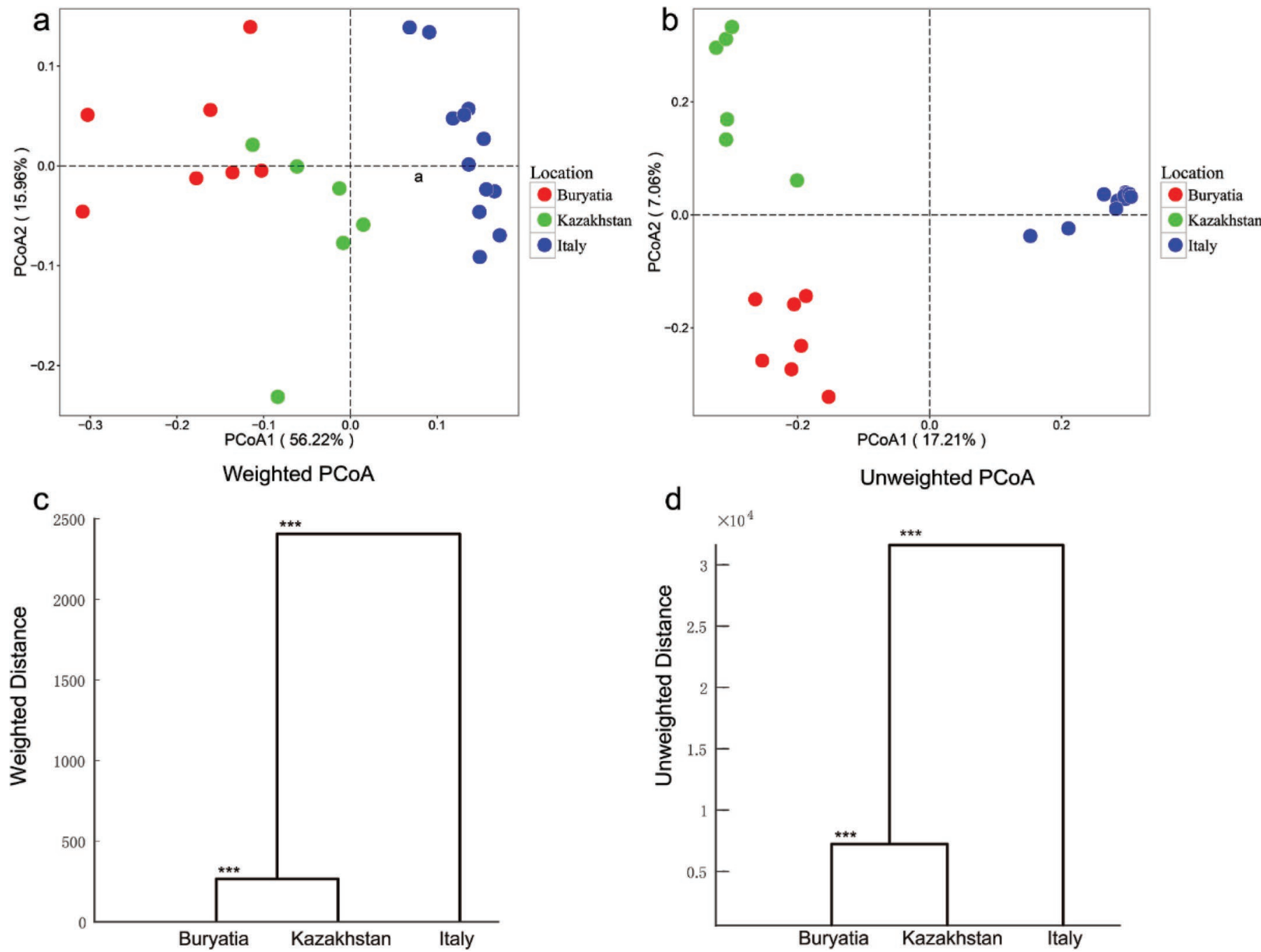

Figure 5. Weighted (a) and unweighted (b) UniFrac principal component analysis (PCoA) score plots based on principal components 1 and 2. Clustering of cheese samples from 3 countries based on (c) weighted and (d) unweighted UniFrac distances calculated using multivariate ANOVA; ${ }^{* * *} P<0.001$. Color version available online 
beverages. Lactobacillus helveticus has high proteolytic capacity, an ability that is linked with the release of bioactive peptides upon cheese and dairy fermentation; proteolysis is a complicated and crucial biochemical process during cheese ripening (Hati et al., 2016). On the other hand, it is worth noting that sequences representing several potential pathogens (namely, $K$. pneumonia, K. oxytoca, and Raoultella ornithinolytica) varied markedly between samples. Klebsiella oxytoca is commonly present in the intestinal tract of animals and humans, soil, and water. It may increase the infection risk in patients that suffer from problems such as bronchopneumonia, urinary infection, and septicemia (Darby et al., 2014; Singh et al., 2016). Raoultella ornithinolytica may cause human infections in rare cases (Drancourt et al., 2001; Kaya et al., 2015). The presence of sequences representing potential pathogens, particularly those that originate from human and animal guts, might reflect poor sanitary conditions during cheese production. The large variation among samples might

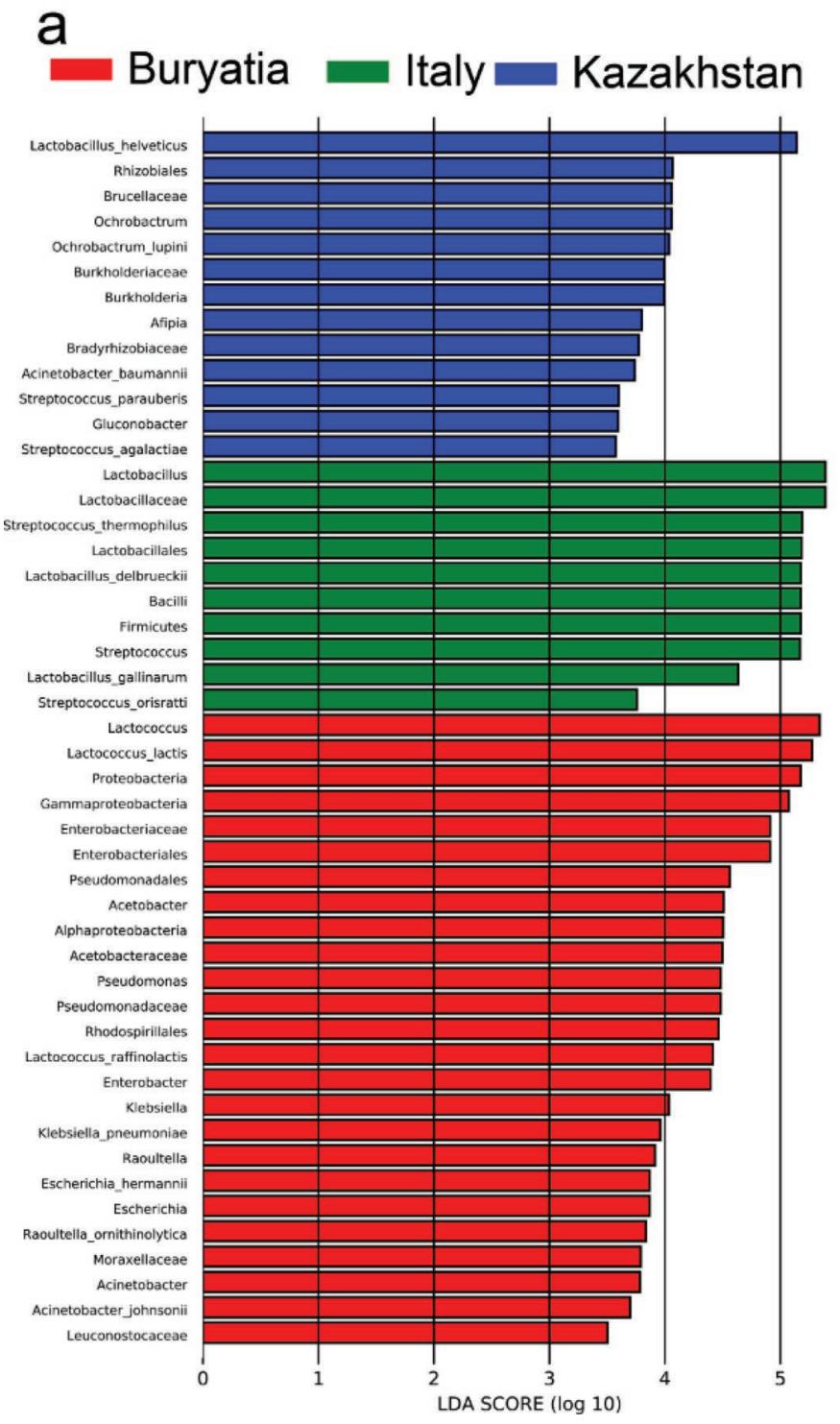

b

\section{Cladogram}

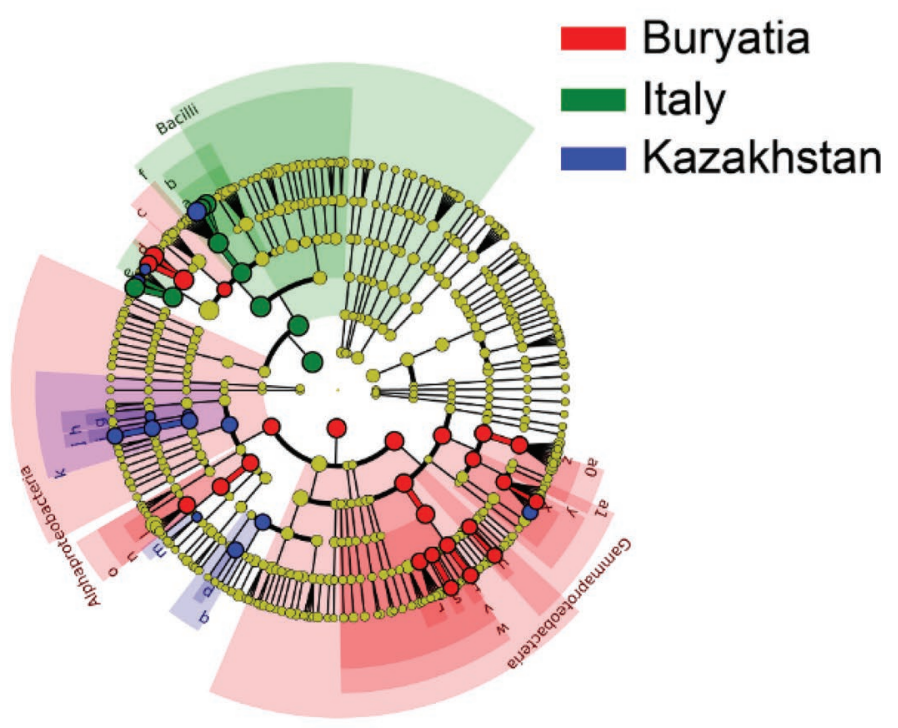

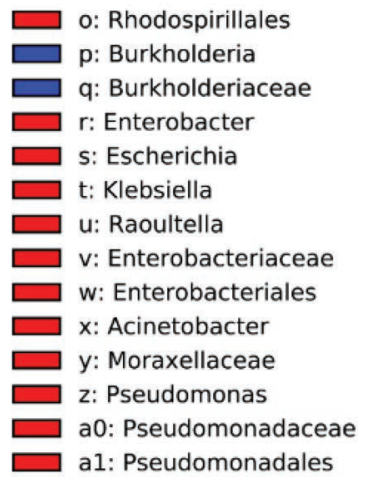

Figure 6. Identification of discriminant taxa between cheeses originating from 3 countries by linear discriminant analysis (LDA) of the effect size. (a) Horizontal bar chart showing discriminant taxa. Significant discriminant taxa of Buryatian, Italian, and Kazakhstan cheeses are represented by red, green, and blue, respectively. (b) Cladogram of the microbiota. Significant discriminant taxon nodes of the Buryatian, Italian, and Kazakhstan cheeses are represented by red, green, and blue, respectively, while nondiscriminant taxon nodes are represented by yellow. Branch areas are shaded according to the highest-ranked variety for that taxon. The LDA score indicates the level of differentiation among cheese samples from the 3 countries. A threshold value of 3.5 was used as the cut-off level. 
indicate different levels of hygiene measures practiced by the households during cheese production.

Our comparisons of cheeses among geographic regions revealed differences between cheeses from Buryatia, Italy, and Kazakhstan. The LDA of the effect size analyses, performed to further pinpoint the differential taxa in these microbiota communities, indicated high levels of most bacterial taxa in Buryatian cheese. Interestingly, both L. raffinolactis and L. lactis could have been used as biomarkers of Buryatian cheese. Zhong et al. studied cheese samples from different provinces in China and found, as we did, that the microbiota communities varied by geographical location (Zhong et al., 2016). Geographical factors include differences in various environmental factors such as altitude, climate, temperature, atmospheric pressure, and sunlight (Sun et al., 2010), which together shape the microbiome of the final fermented product. Furthermore, the microbial communities of cheeses are likely to be influenced by the milk source, milk treatment, and ripening conditions. For instance, buffalo milk has higher levels of solid content, protein, and fat compared with cow milk, resulting in differences in the microbiota structure and composition of cheeses made from the 2 milk sources (Ahmad et al., 2008; Correddu et al., 2017). However, the microbes intrinsically present in the raw milk may have little influence on the microbiota of the final products (Ercolini, 2013; De Filippis et al., 2014). Another important determining factor could be the microorganisms in the cheese-production environment, including those from human skin, ambient air, and processing equipment (Motato et al., 2017). These microorganisms together with the specific conditions and technologies of cheese making would drive ecological succession during various stages of cheese production.

\section{CONCLUSIONS}

We conducted comprehensive analyses of the microbiota of Buryatian cottage cheese using the SMRT platform, and identified and preserved 62 novel LAB strains. The bacterial microbiota differed by region. This study may facilitate future improvement and industrialization of cheese production.

\section{ACKNOWLEDGMENTS}

This research was supported by the China Agriculture Research System (Beijing, China; Grant CARS-36), the National Natural Science Foundation of China (Beijing, China; Grant No. 31601454), and the Excellent Young Scientist Foundation of Inner Mongolia Agricultural University of China (Grant No. 2017XYQ-4).

\section{REFERENCES}

Ahmad, S., I. Gaucher, F. Rousseau, E. Beaucher, M. Piot, J. F. Grongnet, and F. Gaucheron. 2008. Effects of acidification on physico-chemical characteristics of buffalo milk: A comparison with cow's milk. Food Chem. 106:11-17.

Arioli, S., C. Monnet, S. Guglielmetti, C. Parini, I. D. Noni, J. Hogenboom, P. M. Halami, and D. Mora. 2007. Aspartate biosynthesis is essential for the growth of Streptococcus thermophilus in milk, and aspartate availability modulates the level of urease activity. Appl. Environ. Microbiol. 73:5789-5796.

Cole, J. R., B. Chai, R. J. Farris, Q. Wang, A. S. Kulamsyedmohideen, D. M. Mcgarrell, A. M. Bandela, E. Cardenas, G. M. Garrity, and J. M. Tiedje. 2007. The ribosomal database project (RDPII): Introducing myRDP space and quality controlled public data. Nucleic Acids Res. 35:D169-D172.

Correddu, F., J. Serdino, M. G. Manca, G. Cosenza, A. Pauciullo, L. Ramunno, and N. P. P. Macciotta. 2017. Use of multivariate factor analysis to characterize the fatty acid profile of buffalo milk. J. Food Compos. Anal. 60:25-31.

Dalmasso, A., M. D. S. d. Rio, T. Civera, D. Pattono, B. Cardazzo, and M. T. Bottero. 2016. Characterization of microbiota in Plaisentif cheese by high-throughput sequencing. Lebensm. Wiss. Technol. 69:490-496.

Darby, A., K. Lertpiriyapong, U. Sarkar, U. Seneviratne, D. S. Park, E. R. Gamazon, C. Batchelder, C. Cheung, E. M. Buckley, and N. S. Taylor. 2014. Cytotoxic and pathogenic properties of Klebsiella oxytoca isolated from laboratory animals. PLoS One 9:e100542.

De Filippis, F., A. L. Storia, G. Stellato, M. Gatti, and D. Ercolini. 2014. A selected core microbiome drives the early stages of three popular Italian cheese manufactures. PLoS One 9:e89680.

Doyle, J. J. 1987. A rapid DNA isolation procedure for small amounts of fresh leaf tissue. Phytochem. Bull. 19:11-15.

Drancourt, M., C. Bollet, A. Carta, and P. Rousselier. 2001. Phylogenetic analyses of Klebsiella species delineate Klebsiella and Raoultella gen. nov., with description of Raoultella ornithinolytica comb. nov., Raoultella terrigena comb. nov. and Raoultella planticola comb. nov. Int. J. Syst. Evol. Microbiol. 51:925-932.

Ercolini, D. 2013. High-throughput sequencing and metagenomics: Moving forward in the culture-independent analysis of food microbial ecology. Appl. Environ. Microbiol. 79:3148-3155.

Escobar-Zepeda, A., A. Sanchezflores, and B. M. Quirasco. 2016. Metagenomic analysis of a Mexican ripened cheese reveals a unique complex microbiota. Food Microbiol. 57:116-127.

Fernandez, E., A. Alegria, S. Delgado, M. C. Martin, and B. Mayo. 2011. Comparative phenotypic and molecular genetic profiling of wild Lactococcus lactis ssp. lactis strains of the L. lactis ssp. lactis and $L$. lactis ssp. cremoris genotypes, isolated from starter-free cheeses made of raw milk. Appl. Environ. Microbiol. 77:5324-5335.

Gesudu, Q., Z. Yi, X. Xi, C. H. Qiang, H. Xu, W. Huang, H. Zhang, B. Menghe, and W. Liu. 2016. Investigating bacterial population structure and dynamics in traditional koumiss from Inner Mongolia using single molecule real-time sequencing. J. Dairy Sci. 99:7852-7863.

González-Córdova, A. F., C. Yescas, Á. M. Ortiz-Estrada, R.-A. M. L. De, A. Hernández-Mendoza, and B. Vallejo-Cordoba. 2016. Invited review: Artisanal Mexican cheeses. J. Dairy Sci. 99:3250-3262.

Hati, S., A. Sakure, and S. Mandal. 2016. Impact of proteolytic Lactobacillus helveticus MTCC5463 on production of bioactive peptides derived from honey based fermented milk. Int. J. Pept. Res. Ther. $23: 297-303$.

Hou, Q., H. Xu, Y. Zheng, X. Xi, L. Y. Kwok, Z. Sun, H. Zhang, and W. Zhang. 2015. Evaluation of bacterial contamination in raw milk, ultra-high temperature milk and infant formula using single molecule, real-time sequencing technology. J. Dairy Sci. 98:84648472 .

Kamber, U. 2007. The traditional cheeses of Turkey: Cheeses common to all regions. Food Rev. Int. 24:1-38.

Kaya, S., G. Bayramoğlu, M. Sönmez, and İ. Köksal. 2015. Raoultella ornithinolytica causing fatal sepsis. Braz. J. Infect. Dis. 19:230-231. 
Kimoto-Nira, H., R. Aoki, K. Mizumachi, K. Sasaki, H. Naito, T. Sawada, and C. Suzuki. 2012. Interaction between Lactococcus lactis and Lactococcus raffinolactis during growth in milk: Development of a new starter culture. J. Dairy Sci. 95:2176-2185.

Koning, C. J., D. M. Jonkers, E. E. Stobberingh, L. Mulder, F. M Rombouts, and R. W. Stockbrügger. 2008. The effect of a multispecies probiotic on the intestinal microbiota and bowel movements in healthy volunteers taking the antibiotic amoxycillin. Am. J. Gastroenterol. 103:178-189.

Lazzi, C., S. Turroni, A. Mancini, E. Sgarbi, E. Neviani, P. Brigidi, and M. Gatti. 2014. Transcriptomic clues to understand the growth of Lactobacillus rhamnosus in cheese. BMC Microbiol. 14:28.

Li, J., Y. Zheng, H. Xu, X. Xi, Q. Hou, S. Feng, L. Wuri, Y. Bian, Z. $\mathrm{Yu}$, and L. Y. Kwok. 2017. Bacterial microbiota of Kazakhstan cheese revealed by single molecule real time (SMRT) sequencing and its comparison with Belgian, Kalmykian and Italian artisanal cheeses. BMC Microbiol. 17:13.

Litopoulou-Tzanetaki, E., and N. Tzanetakis. 2014. The microfloras of traditional Greek cheeses. Microbiol. Spectr. 2:CM-0009-CM2012.

Lozupone, C., M. Hamady, and R. Knight. 2006. UniFrac-An online tool for comparing microbial community diversity in a phylogenetic context. BMC Bioinformatics 7:371.

Luiz, L. M. P., R. D. Castro, S. H. C. Sandes, J. G. Silva, L. G. Oliveira, G. A. Sales, A. C. Nunes, and M. R. Souza. 2017. Isolation and identification of lactic acid bacteria from Brazilian Minas artisanal cheese. CYTA J. Food 15:125-128.

Lusk, T. S., A. R. Ottesen, J. R. White, M. W. Allard, E. W. Brown, and J. A. Kase. 2012. Characterization of microflora in Latin-style cheeses by next-generation sequencing technology. BMC Microbiol. $12: 254$

Montel, M. C., S. Buchin, A. Mallet, C. Delbes-Paus, D. A. Vuitton, N. Desmasures, and F. Berthier. 2014. Traditional cheeses: Rich and diverse microbiota with associated benefits. Int. J. Food Microbiol. 177:136-154.

Mora, D., and S. Arioli. 2014. Microbial urease in health and disease. PLoS Pathog. 10:e1004472.

Motato, K. E., C. Milani, M. Ventura, F. E. Valencia, P. Ruasmadiedo, and S. Delgado. 2017. Bacterial diversity of the Colombian fermented milk "Suero Costeño" assessed by culturing and highthroughput sequencing and DGGE analysis of 16S rRNA gene amplicons. Food Microbiol. 68:129-136.

Nakano, K., A. Shiroma, M. Shimoji, H. Tamotsu, N. Ashimine, S. Ohki, M. Shinzato, M. Minami, T. Nakanishi, and K. Teruya. 2017. Advantages of genome sequencing by long-read sequencer using SMRT technology in medical area. Hum. Cell 30:149-161.

O'Sullivan, D., R. P. Ross, G. F. Fitzgerald, and A. Coffey. 2000 Investigation of the relationship between lysogeny and lysis of Lactococcus lactis in cheese using prophage-targeted PCR. Appl. Environ. Microbiol. 66:2192-2198.

Price, M. N., P. S. Dehal, and A. P. Arkin. 2010. FastTree 2-Approximately maximum-likelihood trees for large alignments. PLoS One 5:e9490

Quigley, L., O. O'Sullivan, T. P. Beresford, R. P. Ross, G. F. Fitzgerald, and P. D. Cotter. 2012. High-throughput sequencing for detec- tion of subpopulations of bacteria not previously associated with artisanal cheeses. Appl. Environ. Microbiol. 78:5717-5723.

Riquelme, C., S. Câmara, M. L. Dapkevicius, P. Vinuesa, S. C. Da, F. X. Malcata, and O. A. Rego. 2015. Characterization of the bacterial biodiversity in Pico cheese (an artisanal Azorean food). Int. J. Food Microbiol. 192:86-94.

Silvetti, T., E. Capra, S. Morandi, P. Cremonesi, M. Decimo, F. Gavazzi, R. Giannico, I. D. Noni, and M. Brasca. 2017. Microbial population profile during ripening of Protected Designation of Origin (PDO) Silter cheese, produced with and without autochthonous starter culture. Lebensm. Wiss. Technol. 84:821-831.

Singer, E., B. Bushnell, D. Colemanderr, B. Bowman, R. M. Bowers, A. Levy, E. A. Gies, J. F. Cheng, A. Copeland, and H. P. Klenk. 2016. High-resolution phylogenetic microbial community profiling. ISME J. 10:2020-2032.

Singh, L., M. P. Cariappa, and M. Kaur. 2016. Klebsiella oxytoca: An emerging pathogen? Med. J. Armed Forces India 72(Suppl 1):559-561.

Sun, Z., W. Liu, W. Gao, M. Yang, J. Zhang, L. Wu, J. Wang, B. Menghe, T. Sun, and H. Zhang. 2010. Identification and characterization of the dominant lactic acid bacteria from kurut: The naturally fermented yak milk in Qinghai, China. J. Gen. Appl. Microbiol. 56:1-10.

Tsydenova, D. T. 2008. Destiny in the traditional beliefs of the aginsk buryats. Archaeol. Ethnol. Anth. 34:112-121.

Walsh, A. M., F. Crispie, M. J. Claesson, and P. D. Cotter. 2017 Translating omics to food microbiology. Annu. Rev. Food Sci Technol. 8:113-134.

Xu, H., W. Huang, Q. Hou, L. Y. Kwok, Z. Sun, H. Ma, F. Zhao, Y. K. Lee, and H. Zhang. 2017. The effects of probiotics administration on the milk production, milk components and fecal bacteria microbiota of dairy cows. Sci. Bull. 62:767-774.

Yu, J., W. Gao, M. Qing, Z. Sun, W. Wang, W. Liu, L. Pan, T. Sun, H. Wang, and N. Bai. 2012. Identification and characterization of lactic acid bacteria isolated from traditional pickles in Sichuan, China. J. Gen. Appl. Microbiol. 58:163-172.

Yu, J., Z. Sun, W. Liu, J. Zhang, T. Sun, Q. Bao, and H. Zhang. 2009. Rapid identification of lactic acid bacteria isolated from home-made fermented milk in Tibet. J. Gen. Appl. Microbiol. 55:181-190.

Yu, J., H. M. Wang, M. S. Zha, Y. T. Qing, N. Bai, Y. Ren, X. X. Xi, W. J. Liu, B. L. G. Menghe, and H. P. Zhang. 2015. Molecular identification and quantification of lactic acid bacteria in traditional fermented dairy foods of Russia. J. Dairy Sci. 98:5143-5154.

Zheng, Y., X. Xi, H. Xu, Q. Hou, Y. Bian, Z. Yu, L. Y. Kwok, W. Zhang, Z. Sun, and H. Zhang. 2016. Using PacBiol. long-read highthroughput microbial gene amplicon sequencing to evaluate infant formula safety. J. Agric. Food Chem. 64:6993.

Zhong, Z., Q. Hou, L. Kwok, Z. Yu, Y. Zheng, Z. Sun, B. Menghe, and H. Zhang. 2016. Bacterial microbiota compositions of naturally fermented milk are shaped by both geographic origin and sample type. J. Dairy Sci. 99:7832-7841. 